\title{
EVALUATION OF EFFICACY OF DIFFERENT MODALITIES IN TREATMENT OF FRACTURE METATARSAL
}

\author{
SATYAPRATEEK KAUSHIK*, VERMA RK, GUPTA SP, BHAIRWA RAJKUMAR
}

Department of Orthopaedics, Mahatma Gandhi Medical College and Hospital, Jaipur, Rajasthan, India. E-mail: skaushik213@gmail.com Received: 25 February 2021, Revised and Accepted: 01 April 2021

\section{ABSTRACT}

Objectives: This study mainly aims to evaluate the efficacy of three different modalities in treatment of fracture metatarsal.

Methods: A hospital-based prospective comparative study was conducted in Mahatma Gandhi Medical College, Jaipur, from December 2018 to June 2020 , involving 50 patients above 16 years of age presented with isolated metatarsal fractures and grade I open fractures were included in the study. Patients fulfilling the inclusion criteria were then allocated to one of three groups. Group A - Conservative management, Group B - K-wire fixation, and Group C - external Fixator. Outcome measurement was done using AOFAS MID FOOT SCALE (100 points total).

Results: Majority of 29 patients belong to 35-60 years of age group followed by 18 patients in 26-35 years. Maximum number of cases is reported in male category that was 45 cases. Majority of the cases falls under the category of road traffic accidents that are 28 cases which is about $56 \%$ of the total cases. In 37 cases right foot was involved, whereas 13 cases are on the left side, suggesting the right was the dominant side. About $48 \%$ of the second metatarsal and $30 \%$ of the third metatarsal showed the maximum involvement. Maximum number of cases were designated as excellent having score between 90 and 100 that was about 38 cases which are $76 \%$ of total cases suggesting every treatment modality was good in its own right. There was a significant difference between the different treatment modalities at final follow-up $\mathrm{p}<0.05$.

Conclusion: All treatment modalities equally good, achieving good fracture union, decreased incidence of pain, and achieve a good range of movements, but complications rate was more in the conservative group as compared to other groups.

Keywords: Fracture metatarsal, Conservative management, K-wire fixation, External fixator.

(C) 2021 The Authors. Published by Innovare Academic Sciences Pvt Ltd. This is an open access article under the CC BY license (http://creativecommons.org/ licenses/by/4.0/) DOI: http://dx.doi.org/10.22159/ajpcr.2021v14i5.41218. Journal homepage: https://innovareacademics.in/journals/index.php/ajpcr

\section{INTRODUCTION}

Foot is a great dynamic structure of the body; consists of 26 small bones, joints, multiple ligaments and tendons that provide a great stable structure which enables a person to walk, jump, and run even on an uneven surface without any difficulty.

The foot, which is situated at the lower part of human anatomical hierarchy, carries the whole body to its destination is still the one that is mostly neglected by patients and clinicians. Although the various traumas to the foot encountered out of that fracture metatarsal is one of them.

Metatarsal fractures represent $5-6 \%$ of fractures encountered in primary care [1]. They range from easily managed fractures to more complicated fractures that require surgical intervention. With a basic knowledge of metatarsal injuries, primary care physicians can manage selected metatarsal fractures and further identify patients who need a referral.

Contiguous metatarsal fractures were also common and occurred in $9 \%$ of all metatarsal fractures. A total of $60 \%$ of middle metatarsal fractures were associated with a neighboring metatarsal injury. Middle metatarsal fractures are generally neglected because that is secured on the medial side by the first metatarsal and lateral side by the fifth metatarsal that prevents displacement of fracture fragments. Even then, this fracture creates lots of problems and disability during walking and long-standing, for example, stress fracture being one of them. Stress fractures which occur in athletes and military personnel commonly affect the foot skeleton. The proximal shaft of the second, third, and fourth metatarsals are the most frequent sites of stress fractures in human adult bones [2-4]. The fifth metatarsal remains the most common site of injury: Such fractures are almost ten times more common in males and the mean age is 26 years [5]. In high-level athletes, fracture through the base of the fifth metatarsal is the fourth most common foot and ankle injury [6].

While we were dealing with foot injuries, we came up with a lot of problems such as patients arriving late after injury, as a result of which there were lots of neglected foot injury cases present. Another problem being the economic crunch that leads to mismanagement of patients and these patients were unable to receive the proper treatment. In the case of compound fracture, infection is the main culprit which leads to modification of treatment. Hence, the present study was conducted with the aim to evaluate the efficacy of three different modalities in the treatment of fracture metatarsal.

\section{METHODS}

A hospital-based prospective comparative study was conducted in Mahatma Gandhi Medical College, Jaipur, from December 2018 to June 2020 , involving 50 numbers of patients. The study was done after taking due clearance from Clearance from Institute Ethics Committee. Furthermore, written and informed consent of all the patients was taken before their enrolment in the study.

\section{Inclusion criteria}

The following criteria were included in the study:

1. Isolated metatarsal fractures

2. Open fractures grade I

3. Age above 16 years.

\section{Exclusion criteria}

The following criteria were excluded from the study:

1. Grade II and III open fractures 
2. Associated lower limb long bone fractures

3. Age below 16 years.

\section{Protocol}

The management of the patient was started in the emergency room as soon as possible. Early management of these patients was done according to standard ATLS protocol. After primary stabilization, the patient underwent a complete survey which included radiographs of cervical spine, chest abdomen, dorso-lumbar spine, pelvis, and the injured limb with proximal-distal joint. Open injuries were thoroughly irrigated and debrided to remove any foreign body, contamination, grass, dirt, or any devitalized tissue.

After primary management, the patient was checked if he fits in inclusion criteria or not. Written consent was taken in patient's native language. Patients fulfilling the inclusion criteria were then allocated to one of three groups.

\section{Routine investigation}

- $\quad$ CBC, ESR, serum electrolyte

- Random blood sugar, blood urea, serum creatinine

- Blood grouping and cross-matching

- Other investigations depending on the patient's pre-morbid condition.

\section{Groups}

- GROUP A - Conservative management

- GROUP B - K-wire fixation

- GROUP C - External fixator.

Outcome measurement

- AOFAS MID FOOT SCALE (100 points total).

- (AMERICAN ORTHOPAEDIC FOOT AND ANKLE SCORE).

- The surveys include a mixture of questions that are both subjective and objective in nature. The pain category, which asks patients a single question about their level of pain, is subjective, while the alignment category (to be answered by the physician) was objective.

\section{RESULTS}

Table 1, majority 29 patients belong to 35-60 years of age group followed by 18 patients in 26-35 years. Maximum number of cases is reported in male category that was 45 cases, which was about $90 \%$ of total cases and only 5 cases of female are reported, which was only $10 \%$ of total cases. There was hardly any difference, as cases from urban and rural areas are nearly equal consists of $52 \%$ cases from urban areas and $48 \%$ cases from rural areas.

Table 2, maximum number of cases falls under the category of road traffic accidents that were 28 cases which were about $56 \%$ of the total cases followed by 15 cases belongs to fall of heavy object that was $30 \%$ of cases and remaining modes of injury that was twisting get 5 cases which were $10 \%$ of total cases while assault got only 2 cases which $4 \%$ of total cases. Side of injury does not have much significance but still in 37 cases, the right foot is involved which was $74 \%$ of cases, whereas 13

Table 1: Demographic details of the study population

\begin{tabular}{lll}
\hline Variables & Number of cases & Percentage \\
\hline Age in years & 3 & \\
18-25 & 18 & 6.00 \\
$26-35$ & 29 & 36.00 \\
$36-60$ & & 58.00 \\
Sex & 45 & \\
Male & 5 & 90 \\
Female & & 10 \\
Area of residence & 24 & 48.00 \\
Rural & 26 & 52.00 \\
Urban & 50 & 100 \\
Total & &
\end{tabular}

cases are of the left side which was $26 \%$ of total cases suggesting right was the dominant side.

Table 3, a total percentage that is $48 \%$ of the second metatarsal and $30 \%$ of the third metatarsal showed the maximum involvement. While the fourth and fifth metatarsal consists of $20 \%$ and $18 \%$, respectively, and remaining first metatarsal with only $6 \%$ involvement.

Table 4, every patient was given a set of questionnaires according to it, patient was assigned to a score between 0 and 100 . It was been calculated at the time of injury, at 2 weeks, 2 months, and 6 months. As expected, patients that were receiving treatment have increment in mean score being lowest at the time of injury that was 42.68 which increases at follow-up that was 69.82 at 2 weeks, 78.24 at 2 months, and 87.88 at 6 months. Maximum number of cases were designated as excellent having score between 90 and 100 that was about 38 cases which were $76 \%$ of total cases suggesting every treatment modality was good in its own right. However, excellent cases do complain of pain-causing limping during walking and are not fully satisfied. Rest 7 cases were designated as good, consisting of $14 \%$ of total cases and 5 cases were designated as fair consisting of $10 \%$ of total cases.

Table 5, comparison between conservative management and k-wire was done. Statistical significance has been calculated at follow-up that is at 2 weeks, 2 months, and 6 months which was $0.002,0.006$, and 0.014 . Similarly, comparison between conservative management and external fixation has been done. Statistical significance has been calculated at follow-up that was at 2 weeks, 2 months, and 6 months which was $0.027,0.045$, and 0.035 . Comparison between k-wire and external fixation has been done. Statistical significance has been calculated at follow-up that was at 2 weeks, 2 months, and 6 months which is $0.04,0.12$, and 0.006 . Suggesting there was

Table 2: Distribution according to mode of injury and laterality

\begin{tabular}{lll}
\hline Variables & Number of cases & Percentage \\
\hline Mode of injury & & \\
RTA & 28 & 56.00 \\
Fall of object & 15 & 30.00 \\
Twist & 5 & 10.00 \\
$\quad$ Assault & 2 & 4.00 \\
Side involved & & \\
Right & 37 & 74.00 \\
Left & 13 & 26.00 \\
Total & 50 & 100.00 \\
\hline
\end{tabular}

Table 3: Distribution according to metatarsals involved

\begin{tabular}{lll}
\hline Metatarsals involved & Number of cases & Percentage \\
\hline 1 & 3 & 6.00 \\
2 & 24 & 48.00 \\
3 & 15 & 30.00 \\
4 & 10 & 20.00 \\
5 & 9 & 18.00 \\
\hline
\end{tabular}

Table 4: Mean AOFAS score and outcome in the study population

\begin{tabular}{ll}
\hline Interval & Mean \pm SD \\
\hline AOFAS score & \\
Before treatment & $42.68 \pm 14.43$ \\
2 week & $69.82 \pm 8.10$ \\
2 month & $78.24 \pm 8.69$ \\
6 month & $87.88 \pm 10.11$ \\
& $n(\%)$ \\
Outcome & $7(14.0)$ \\
Good & $5(10.0)$ \\
Fair & $38(76.0)$ \\
Excellent &
\end{tabular}


Table 5: Comparison of treatment outcome between the groups at different time intervals

\begin{tabular}{|c|c|c|c|c|c|c|c|}
\hline \multirow[t]{2}{*}{ Treatment } & \multirow{2}{*}{$\begin{array}{l}\text { Number } \\
\text { of cases }\end{array}$} & \multicolumn{2}{|c|}{2 Week } & \multicolumn{2}{|c|}{2 Months } & \multicolumn{2}{|c|}{6 Months } \\
\hline & & Mean & SD & Mean & SD & Mean & SD \\
\hline Conservative & 27 & 72.19 & 2.75 & 81.92 & 3.32 & 91.59 & 3.99 \\
\hline K wire & 19 & 66.84 & 12.14 & 75.53 & 11.91 & 84.26 & 14.14 \\
\hline $\begin{array}{l}\text { External } \\
\text { fixator }\end{array}$ & 04 & 68.00 & 3.27 & 75.75 & 3.86 & 80.00 & 6.32 \\
\hline \multicolumn{2}{|c|}{ (Conservative vs. } & \multicolumn{2}{|l|}{$0.002^{*}$} & \multicolumn{2}{|l|}{$0.006^{*}$} & \multicolumn{2}{|l|}{$0.014^{*}$} \\
\hline \multicolumn{2}{|c|}{$\begin{array}{l}\text { (Conservative vs. } \\
\text { external fixator) }\end{array}$} & \multicolumn{2}{|l|}{$0.027^{*}$} & \multicolumn{2}{|l|}{$0.045^{*}$} & \multicolumn{2}{|l|}{$0.035^{*}$} \\
\hline $\begin{array}{l}\text { p-value } \\
\text { (K-wire vs. ex } \\
\text { fixator) p-valu }\end{array}$ & ternal & \multicolumn{2}{|l|}{$0.040^{*}$} & \multicolumn{2}{|l|}{0.120} & \multicolumn{2}{|l|}{$0.006^{*}$} \\
\hline
\end{tabular}

Test applied: unpaired t-test. ${ }^{*}$ Indicates statistical significance

a significant difference between the different treatment modalities at final follow-up.

\section{DISCUSSION}

Patients with metatarsal fractures often present to primary care settings and present with a variety of situations. Ranging from the relatively benign, isolated central metatarsal fracture to the crush injury leading to extensive damage of the soft tissue and osseous component, these fractures can cause a significant inconvenience to the patient. With the exception of the fifth metatarsal fractures, little standardization was available for the treatment of metatarsal fractures.

The importance of the first metatarsal with regard to overall foot function makes anatomic alignment paramount. Attention to detail can help prevent long-term sequelae and deformities. Central metatarsal fractures have a high chance of union, with little known about extensive complications. However, disruption in the metatarsal parabola can cause undue discomfort. The fifth metatarsal was by far the most common metatarsal that was fractured. A large percentage of this fracture was amenable to conservative treatment. When surgical intervention was implemented, the patient and surgeon can expect a good outcome.

This sample size was very small as well as duration of the study is not too long. Looking with this scenario in our study also has the same condition more and less. This comes out with overall observation even. Better results can be achieved after a long study and a larger sample size.

In this study, a total number of 50 cases have been taken out of which 27 patients underwent conservative management and 19 underwent K-wire fixation and 4 patients underwent external fixation.

Our case series, we considered age from 18 to 60 years and divided into 3 groups. The first group has age between 18 and 25 consist of only 3 cases that are $6 \%$ of total cases. The second group has age between 26 and 35 consist of 18 cases that are $36 \%$ of total cases. Moreover, the third group has age between 36 and 60 consist of 29 cases that are $58 \%$ of total cases. It indicated maximum number of cases falls in the second and third group that is $94 \%$ of total cases; pointing toward the working class that sustains this type of injury. Chandran et al. [7] and Thompson et al. [8] also documented a similar incidence.

In sex distribution, maximum number of cases was reported in male category that was 45 cases; which was about $90 \%$ of total cases and only 5 cases of female were reported, which was only $10 \%$ of total cases. This suggests in Indian scenario, males were dominantly working-class and sustains more traumas

Chandran et al. [7] also have incidence similar to our study with male $90 \%$ and female $10 \%$. The incidence of female patients was significantly higher in Thompson et al. [8] study, which was due to similar working class of male and female in foreign countries.

In area distribution, there was hardly any difference, as cases from urban and rural areas were nearly equal consists of $52 \%$ cases from urban areas and $48 \%$ cases from rural areas. As road traffic accident and industrial machine injury were more common in urban areas.

In our study, the most common mode of injury was high-velocity road traffic accidents accounts for $56 \%$ followed by fall of heavy object $(30 \%)$. The incidence of injury due to RTA was almost similar to series of Gotha et al. [9] and Chandran et al. [7]. The reason of high incidence of RTA can be attributed to better road facilities and technological advancements with an increase number of vehicles and ignorance of traffic rules. Assault and fall of heavy object were other causes.

Incidence of right side metatarsal involvement was significantly higher $(74 \%)$ than the left side $(26 \%)$ in our series as observed by Gotha et al. [9] that right foot commonly involve that left foot further supporting the evidence. As right was the dominant side.

In our series, most common metatarsal involved was second (48\%) followedbythird(30\%).Whilethefourthandfifthmetatarsal involvementwas $20 \%$ and $18 \%$, respectively. Remaining the first metatarsal with only $6 \%$ involvement. Baumfeld et al. [10] study was almost similar involvement of second metatarsal fracture (57\%). Incidence of fifth metatarsal fracture significantly higher in Sarpong et al. [11]

As the study progresses, we encountered various complications such as infection, malunion, and deformity. Of these, malunion and deformity were mostly present in conservative management group as these patients often remove cast of their own and loss of follow-up leading to mismanagement and leads to complications. Six cases have suffered from malunion and in 5 cases, persistence of deformity has been seen. Kim et al. [12] stated that more than 10 degree of displacement in the sagittal plane and 3-4 mm of translation in any plane should be treated surgically as due to the presence of angulations and these cases opted for conservative management, as a result of which leads to malunion and deformities.

Infection rates were higher in operative cases, in which 3 cases of external fixation got infected and 2 cases of $\mathrm{k}$-wire fixation got infected and persistence of deformity was seen in only one case of k-wire fixation. Baumfeld et al. [10] concluded that percutaneous antegrade $\mathrm{k}$-wire surgical treatment was an effective alternative to other types of treatment for lateral metatarsal fractures, with a lower incidence of complications. In our study also, the overall complication rate was found to be lower in K-wire group.

In our series, final outcome was based on AOFAS score. Patients that were treated as conservatively AOFAS score excellent (91.59 out of 100). Patients treated with k-wire fixation and external fixator AOFAS score was good (84.26 and 80.00, respectively). A similar study was conducted by Cakir et al. [13] reported that the general outcome of metatarsal fracture was satisfactory. Moreover, Spector et al. [14] treated 12 patients; 3 with a non-displaced proximal metatarsal fracture, 1 with a displaced proximal metatarsal fracture, 7 with a distal metatarsal fracture, and 1 with a midshaft metatarsal fracture. Five of these patients were treated conservatively without reduction, 2 by anatomic open reduction with internal fixation, and 5 by non-anatomic open reduction with internal fixation. Good to excellent results were obtained in $8(67 \%)$ and fair results in 4 (33\%).

In the case study of Kim et al. [12] conducted a study on k- wire fixation done on 35 patients and the result found excellent with average AOFAS score of 96.7 .

While we were going through literature, it was found most of the authors are treated conservative only or operative only. There are 
few studies who tried to compare between different modalities, but they were not satisfied as conditions were not equal in both the treatment.

Various factors were considered before assigning the patient into a certain group such as low socio-economic conditions of the patients, social dogma in society regarding operative procedures, simply not willing for surgical correction irrespective of severity of injury, and lastly those who were not fit for surgery underwent conservative management. While the educated people who are from a good socioeconomic background who understood the situation underwent fixation through k-wire fixation and external fixation on the basis of severity of injury. AOFAS score was excellent in the conservative group in compare with the operative group due to the larger sample size and have a less severe type of injury.

\section{CONCLUSION}

In our study, metatarsal fractures treated with conservative management with casting, Kirschner wire stabilization and external fixation. AOFAS score was used to assess the functional outcome in our study. At the end of the study, AOFAS score was maximum in conservative groups pointing toward the fact that maximum cases opted for conservative management and least for external fixation. All treatment modalities equally good, achieving good fracture union, decreased incidence of pain, and achieve good range of movements but complications rate is more in the conservative group as compared to other groups. Even though we have maximum complications in the conservative group, we have achieved good union and excellent functional outcome at the end of follow-up.

\section{AUTHORS' CONTRIBUTIONS}

I would like to express my heartiest gratitude to the participants who gave the willingness to participate in the study.

\section{CONFLICTS OF INTEREST}

There are no conflicts of interest.

\section{AUTHORS' FUNDING}

Self.

\section{REFERENCES}

1. Kalia V, Fishman EK, Carrino JA, Fayad LM. Epidemiology, imaging, and treatment of Lisfranc fracture-dislocations revisited. Skeletal Radiol 2012;41:129-36.

2. Geyer M, Sander-Beuermann A, Wegner U, Wirth CJ. Stress reactions and stress fractures in the high performance athlete. Causes, diagnosis and therapy. Unfallchirurg 1993;96:66-74.

3. Harrington T, Crichton KJ, Anderson IF. Overuse ballet injury of the base of the second metatarsal. A diagnostic problem. Am J Sports Med 1993;21:591-8.

4. O'Malley MJ, Hamilton WG, Munyak J. Fractures of the distal shaft of the fifth metatarsal. "Dancer's fracture". Am J Sports Med 1996;24:240-3.

5. Shuen WM, Boulton C, Batt ME, Moran C. Metatarsal fractures and sports. Surgeon 2009;7:86-8.

6. Brockwell J, Yeung Y, Griffith JF. Stress fractures of the foot and ankle. Sports Med Arthrosc Rev 2009;17:149-59.

7. Chandran P, Puttaswamaiah R, Dhillon MS, Gill SS. Management of complex open fracture injuries of the midfoot with external fixation. J Foot Ankle Surg 2006;45:308-15

8. Thompson P, Patel V, Fallat LM, Jarski R. Surgical management of fifth metatarsal diaphyseal fractures: A retrospective outcomes study. J Foot Ankle Surg 2017;56:463-7.

9. Gotha HE, Lareau CR, Fellars TA. Diagnosis and management of lisfranc injuries and metatarsal fractures. R I Med J (2013) 2013;96:33-6.

10. Baumfeld D, Macedo BD, Nery C, Esper LE, Filho MA. Anterograde percutaneous treatment of lesser metatarsal fractures: Technical description and clinical results. Rev Bras Ortop 2015;47:760-4.

11. Sarpong NO, Swindell HW, Trupia EP, Vosseller JT. Metatarsal fractures. J Foot Ankle Orthop 2018;3:2473011418775094.

12. Kim HN, Park YJ, Kim GL, Park YW. Closed antegrade intramedullary pinning for reduction and fixation of metatarsal fractures. J Foot Ankle Surg 2012;51:445-9.

13. Cakir H, Van Vliet-Koppert ST, Van Lieshout EM, De Vries MR, Van Der Elst M, Schepers T. Demographics and outcome of metatarsal fractures. Arch Orthop Trauma Surg 2011;131:241-5.

14. Spector FC, Karlin JM, Scurran BL, Silvani SL. Lesser metatarsal fractures. Incidence, management, and review. J Am Podiatry Assoc 1984;74:259-64. 